\title{
Discrete Chaos in Fractional Henon Map
}

\author{
Tongchun Hu \\ Department of Public Teaching, Hangzhou Polytechnic, Hangzhou, China \\ Email: hutongchun888@126.com
}

Received 21 May 2014; revised 28 June 2014; accepted 12 July 2014

Copyright () 2014 by author and Scientific Research Publishing Inc.

This work is licensed under the Creative Commons Attribution International License (CC BY).

http://creativecommons.org/licenses/by/4.0/

(c) (i) Open Access

\begin{abstract}
In this study, a discrete fractional Henon map is proposed in the Caputo discrete delta's sense. The results show that the discrete fractional calculus is an efficient tool and the maps derived in this way have simpler forms but hold rich dynamical behaviors.
\end{abstract}

\section{Keywords}

Fractional Henon Map, Bifurcation, Difference Scheme, Chaos

\section{Introduction}

The chaotic behavior is one important aspect of dynamical systems. Much attention has been paid to the topic on fractional differential equations in the past decades [1]-[6]. Recently, the chaotic fractional difference systems start to attract increasing attention due to its potential applications in secure communication and control process. As we all know, the difference models can reveal the nonlinear phenomenon more accurately since their structure is of discrete or discontinuous dynamics.

Generally speaking, the map $x_{n+1}=f\left(x_{n}\right)$ does not have any memory, as the state $x_{n+1}$ only depends on $x_{n}$. The memory means that the discrete state $x_{n+1}$ explicitly depends on the previous values $x_{n}, x_{n-1}, \cdots, x_{1}$. There are many methods designed for the fractional difference models to prove that the discrete fractional calculus is an efficient tool to discrete the chaotical systems with a memory effect [7]-[9]. Recently Xiao, Li, et al. [10] focused on chaotification of the derived fractional difference maps by using the practical controllers and computed the Lyapunov exponents of the controlled fractional difference maps. Wu and Baleanu [11] [12] concentrated on applications of the discrete fractional calculus on an arbitrary time scale and used the theories of delta difference equations to reveal the discrete chaos behavior. The results showed that the Caputo discrete delta's sense has simpler forms but effective. Tarasov and Edelman [13] demonstrated how the attractors of fractional maps were different from the attractors of the dissipative standard map and stated that the evolution is depended on all past states with the weight functions.

This work looks similar, but the essential difference is that it adopts different definitions of fractional differ- 
ence. In our research, on the basis of the Caputo-like delta difference [8] [11] [12], we obtain the fractionalized Henon map. The calculation results show that the discrete fractional calculus is an efficient tool and the maps derived in this way have simpler forms but hold rich dynamical behaviors. The remainder of this paper is organized as follows. Section 2 introduces the definitions and the properties of the discrete fractional calculus. Section 3 presents the fractional odd logistic map on time scales and shows the discrete chaotic solutions while the difference orders and the coefficients are changing. Section 4 is the conclusion.

\section{Preliminaries}

Concerning nonlinear fractional differential equation of the form

$$
{ }_{C} D_{0, t}^{\alpha} x(t)=f(t, x(t)), x^{(k)}(0)=x_{0}^{(k)}, \quad k=0,1, \cdots,\lceil\alpha\rceil-1,
$$

here $n=\lceil\alpha\rceil-1$ is the first integer not less than $\alpha$ and ${ }_{C} D_{0, t}^{\alpha}$ is Caputo fractional derivative. It is well known that the initial value problem (1) is equivalent to the Volterra integral system [4]-[7] [10]-[12],

$$
x(t)=\sum_{k=0}^{\lceil\alpha\rceil-1} x_{0}^{(k)} \frac{t^{k}}{k !}+\frac{1}{\Gamma(\alpha)} \int_{0}^{t}(t-\tau)^{\alpha-1} f(\tau, x(\tau)) \mathrm{d} \tau
$$

in the sense that if a continuous function solves (2) if and only if it solves (1).

Considering the discrete fractional calculus, we can get the corresponding fractional difference equation. We start with some necessary definitions from discrete fractional calculus theory and preliminary results so that this paper is self-contained.

Definition 1. (See [10].) Let $v$ th fractional sum of $f$ is defined by

$$
\Delta_{a}^{-v} u(t):=\frac{1}{\Gamma(v)} \sum_{s=a}^{t-v}(t-\sigma(s))^{(v-1)} u(s),
$$

where $a$ is the starting point, $\sigma(s)=s+1$ and $u$ is defined for $s=a \bmod (1)$ and $\Delta_{a}^{-v} u(t)$ is defined for $t=(a+v) \bmod (1)$. In particular $\Delta_{a}^{-v}$ maps a functions defined on $N_{a}$ to functions defined on $N_{a+v}$, where $N_{a}=a, a+1, a+2, \cdots$. In addition,

$$
t^{v}=\frac{\Gamma(t+1)}{\Gamma(t+1-v)}
$$

Definition 2. (See [11].) For $\alpha>0, u: N_{a} \rightarrow R$ and $\alpha$ be given, the Caputo-like delta difference is defined by

$$
{ }_{c} \Delta_{v}^{\alpha} u(t):=\Delta_{a}^{n-v} \Delta^{n} u(t)=\frac{1}{\Gamma(n-v)} \sum_{s=a}^{t-(n-v)}(t-\sigma(s))^{(n-v-1)} u(s), t \in N_{a+n-v}, n=[v]+1,
$$

where $v$ is the difference order.

Theorem 1. (See [12].) For the delta fractional difference equation

$$
\begin{gathered}
{ }_{c} \Delta_{a}^{\alpha} u(t)=f(t+\alpha-1, u(t+\alpha-1)), \\
\Delta^{k} u(a)=u_{k}, n=[\alpha]+1, k=0,1, \cdots, n-1 .
\end{gathered}
$$

the equivalent discrete integral equation can be obtained as

$$
u(t)=u(0)+\frac{1}{\Gamma(\alpha)} \sum_{a+n-\alpha}^{t-\alpha}(t-\sigma(s))^{(\alpha-1)} f(s+\alpha-1, u(s+\alpha-1)),
$$

where the initial iteration reads

$$
u(0)=\sum_{k=0}^{n-1} \frac{(t-a)^{k}}{k !} \Delta^{k} u(a) .
$$

The complex difference equation with long-term memory is obtained here. It can reduce to the classical one when the difference order $\alpha=1$. 


\section{Fractional Henon Map}

The Henon map is given by the following pair of first-order difference equations

$$
\left\{\begin{array}{l}
x_{n+1}=y_{n}+1-\alpha x_{n}^{2} \\
y_{n+1}=\beta x_{n}
\end{array}\right.
$$

where $\alpha$ and $\beta$ are (positive) bifurcation parameters, and the Henon map is the most general two-dimensional quadratic map with the property that the contraction is independent of $x$ and $y$. We can rewrite above equation:

$$
\left\{\begin{array}{l}
\nabla x_{n+1}=y_{n}+1-\alpha x_{n}^{2}-x_{n} \\
\nabla y_{n+1}=\beta x_{n}-y_{n}
\end{array}\right.
$$

From the discrete fractional calculus, we modify the standard map as a fractional one

$$
\left\{\begin{array}{l}
{ }_{c} \Delta_{a}^{\mu} x(t)=y(t+\mu-1)+1-\alpha x(t+\mu-1)^{2}-x(t+\mu-1), \\
{ }_{c} \Delta_{a}^{\mu} y(t)=\beta x(t+\mu-1)-y(t+\mu-1) .0<\mu \leq 1, t \in N_{a+1-\mu} .
\end{array}\right.
$$

From (3), we can obtain the following discrete integral form from $0<\mu \leq 1$,

$$
u(t)=u(a)+\frac{1}{\Gamma(\mu)} \sum_{a+1-\mu}^{t-\mu}(t-\sigma(s))^{(\mu-1)} f(s+\mu-1, u(s+\mu-1)), t \in N_{a+1},
$$

where $\frac{(t-\sigma(s))^{(\mu-1)}}{\Gamma(\mu)}$ is a discrete kernel function and $(t-\sigma(s))^{(\mu-1)}=\frac{\Gamma(t-s)}{\Gamma(t-s+1-\mu)}$. As a result, the numerical formula can be presented explicitly. As a result, the numerical formula can be presented explicitly

$$
u(n)=u(a)+\frac{1}{\Gamma(\mu)} \sum_{1}^{n} \frac{\Gamma(n-j+\mu)}{\Gamma(n-j+1)} f(j-1, u(j-1))
$$

For the fractional Henon map, an explicit numerical formula can be given as

$$
\left\{\begin{array}{l}
x(n+1)=x(a)+\frac{1}{\Gamma(\mu)} \sum_{j=1}^{n} \frac{\Gamma(n-j+\mu)}{\Gamma(n-j+1)}\left(y_{n}+1-a x_{n}^{2}-x_{n}\right) \\
y(n+1)=y(a)+\frac{1}{\Gamma(\mu)} \sum_{j=1}^{n} \frac{\Gamma(n-j+\mu)}{\Gamma(n-j+1)}\left(\beta x_{n}-y_{n}\right),
\end{array}\right.
$$

when $v=1$ the above numerical system is the classical one. Using the numerical formula (6), set the step size $\triangle \alpha=0.002$ and the bifurcation diagrams are plotted in Figure 1. For the classical map, an initial point

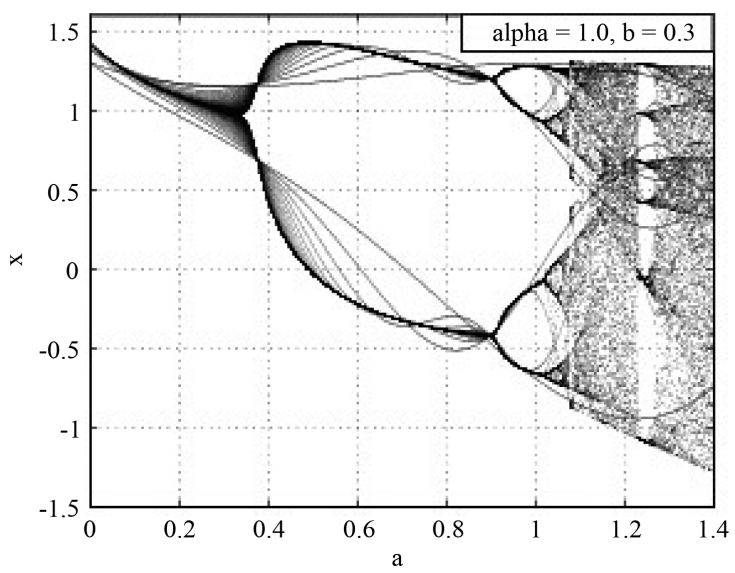

Figure 1. The bifurcation diagram for fractional discrete Henon map when $\alpha=1, x(0)=0, y(0)=0$. 
$x_{0}=0, y_{0}=0, \alpha=1, \beta=0.3$ of the plane will approach a set of points known as the Henon strange attractor, see Figure 2.

Using the numerical formula (6), set the step size $\Delta \alpha=0.002$ and different fractional difference order $\mu$, the bifurcation diagrams are plotted in Figures 3-6. We can readily obtain the intervals of $\mu$ where the chaos

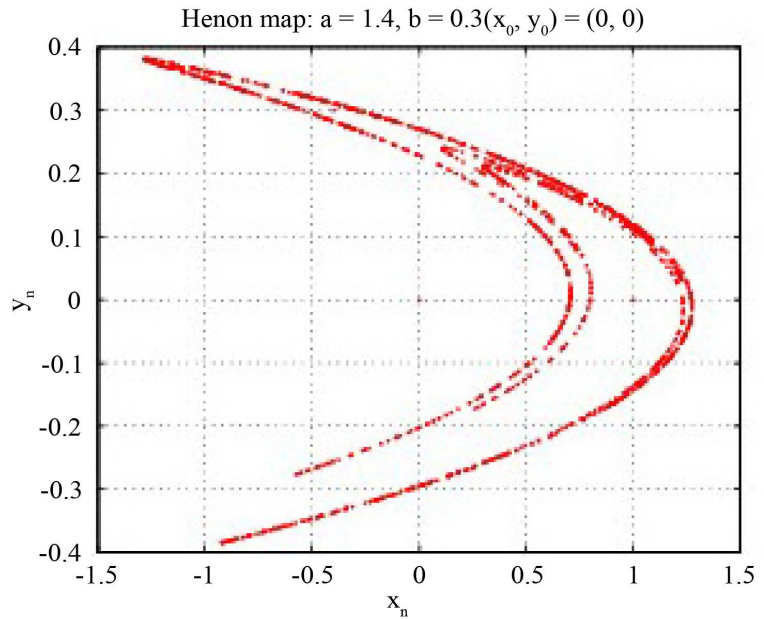

Figure 2. Henon strange attractor for fractional discrete Henon map when $\alpha=1, x(0)=0$.

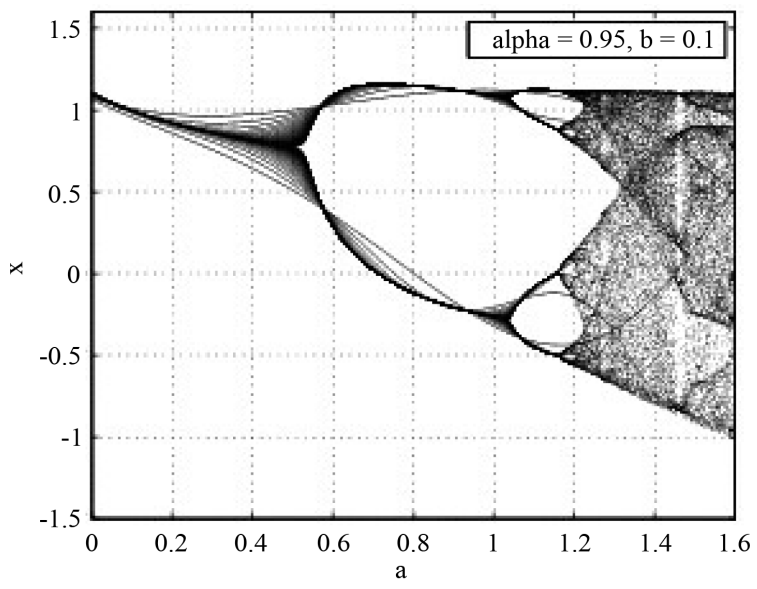

Figure 3. The bifurcation diagram of the fractional discrete Henon map when $\mu=0.95$.

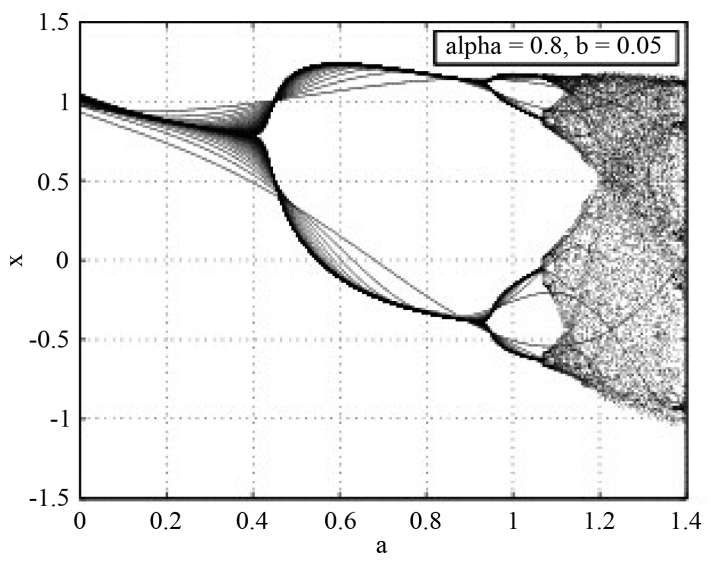

Figure 4. The bifurcation for fractional discrete Henon map diagram when $\mu=0.8$. 


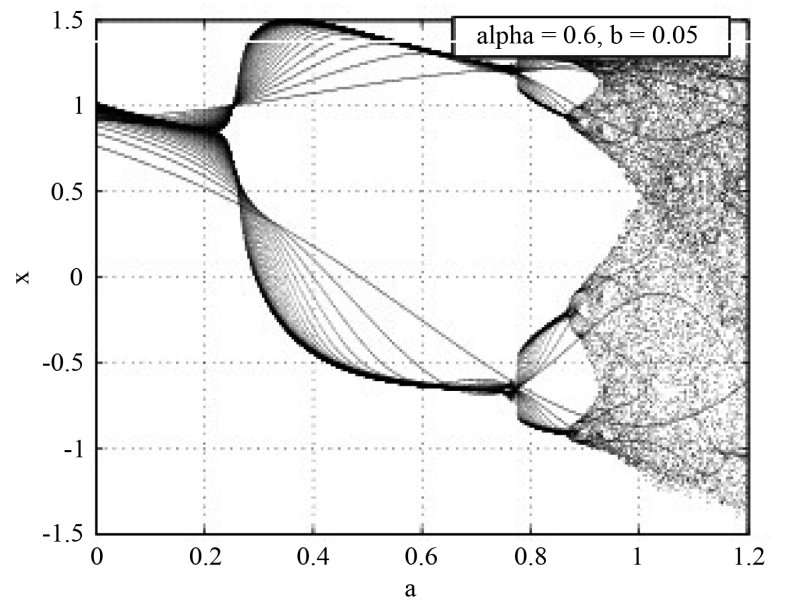

Figure 5. The bifurcation diagram for fractional discrete Henon map when $\mu=0.6$.

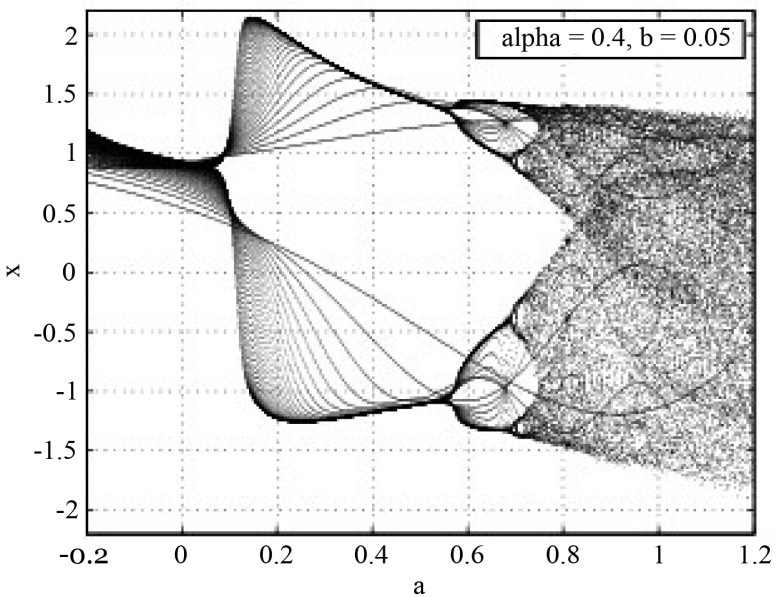

Figure 6. The bifurcation for fractional discrete Henon map diagram when $\mu=0.4$.

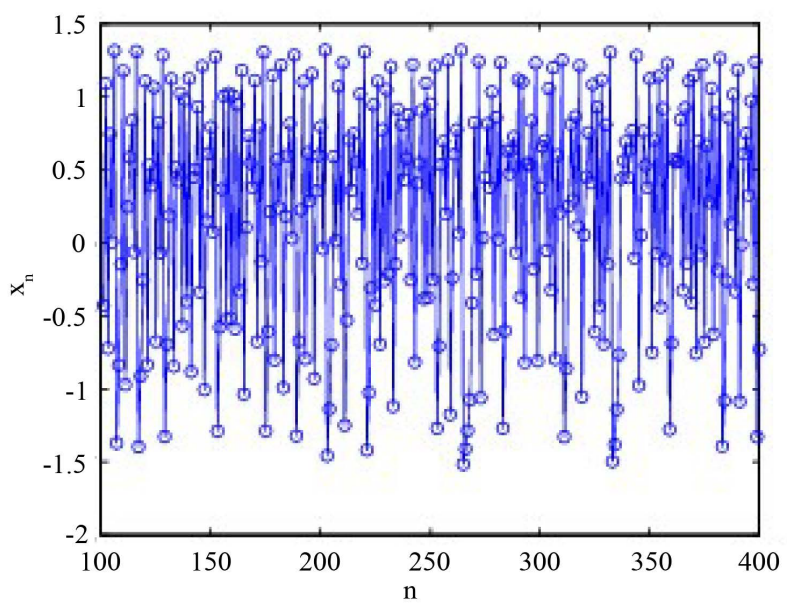

Figure 7. Chaos of the fractional discrete Henon map when $\mu=0.1$.

happens. It can be concluded that the chaos zones are clearly different when we change the difference order $\mu$, moreover, when $\mu=0.1$, the chaos still happened, see Figure 7 . 


\section{Conclusion}

In this paper, the suggested fractional Henon map demonstrates a chaotic behavior with a new type of attractors. The interesting property of the fractional map is the long term memory. Computer simulations of the fractional discrete maps with memory prove that the nonlinear dynamical systems, which are described by the equations with Caputo discrete delta's sense, exhibit a new type of chaotic motion. Through a discrete fractional Henon map it reveals that the dynamical behavior holds the discrete memory even the difference order is very small.

\section{Acknowledgements}

The author thanks the referee for providing constructive correction suggestions. This work is financially supported by the Zhejiang Natural Science Foundation (Grant no. LQ12A01010) and Hangzhou Polytechnic (KZYZ-2009-2).

\section{References}

[1] Hartley, T.T. and Lorenzo, C.F. (2002) Dynamics and Control of Initialized Fractional-Order Systems. Nonlinear Dynamics, 29, 201-233. http://dx.doi.org/10.1023/A:1016534921583

[2] Hwang, C., Leu, J.F. and Tsay, S.Y. (2002) A Note on Time-Domain Simulation of Feedback Fractional-Order Systems. IEEE Transactions on Automatic Control, 47, 625-631. http://dx.doi.org/10.1109/9.995039

[3] Podlubny, I., Petras, I., Vinagre, B.M., O’Leary, P. and Dorcak, L. (2002) Analogue Realizations of Fractional-Order Controllers. Nonlinear Dynamics, 29, 281-296. http://dx.doi.org/10.1023/A:1016556604320

[4] Babakhani, A., Baleanu, D. and Khanbabaie, R. (2012) Hopf Bifurcation for a Class of Fractional Differential Equations with Delay. Nonlinear Dynamics, 29, 721-729. http://dx.doi.org/10.1007/s11071-011-0299-5

[5] Baleanu, D., Diethelm, K., Scalas, E. and Trujillo, J.J. (2012) Fractional Calculus Models and Numerical Methods. World Scientific, Boston.

[6] Li, C.P. and Peng, P.J. (2004) Chaos in Chen’s System with a Fractional Order. Chaos Solitons \& Fractals, 22, 443450. http://dx.doi.org/10.1016/j.chaos.2004.02.013

[7] Atici, F.M. and Eloe, P.W. (2009) Initial Value Problems in Discrete Fractional Calculus. Proceedings of the American Mathematical Society, 137, 981-989. http://dx.doi.org/10.1090/S0002-9939-08-09626-3

[8] Abdeljawad, T. (2011) On Riemann and Caputo Fractional Differences. Computers \& Mathematics with Applications, 62, 1602-1611. http://dx.doi.org/10.1016/j.camwa.2011.03.036

[9] Chen, F.L., Luo, X.N. and Zhou, Y. (2011) Existence Results for Nonlinear Fractional Difference Equation. Advances in Difference Equations, 2011, Article ID: 713201. http://dx.doi.org/10.1155/2011/713201

[10] Xiao, H., Ma, Y. and Li, C.P. (2014) Chaotic Vibration in Fractional Maps. Journal of Vibration and Control, 20, 964972. http://dx.doi.org/10.1177/1077546312473769

[11] Wu, G.C., Baleanu, D. and Zeng, S.D. (2014) Discrete Chaos in Fractional Sine and Standard Maps. Physics Letters A, 378, 484-487. http://dx.doi.org/10.1016/j.physleta.2013.12.010

[12] Wu, G.C. and Baleanu, D. (2014) Discrete Chaos in Fractional Delayed Logistic Maps. Nonlinear Dynamics, in Press.

[13] Tarasov, V.E. and Edelman, M. (2010) Fractional Dissipative Standard Map. Chaos, 20, Article ID: 02327. http://dx.doi.org/10.1063/1.3443235 
Scientific Research Publishing (SCIRP) is one of the largest Open Access journal publishers. It is currently publishing more than 200 open access, online, peer-reviewed journals covering a wide range of academic disciplines. SCIRP serves the worldwide academic communities and contributes to the progress and application of science with its publication.

Other selected journals from SCIRP are listed as below. Submit your manuscript to us via either submit@scirp.org or Online Submission Portal.
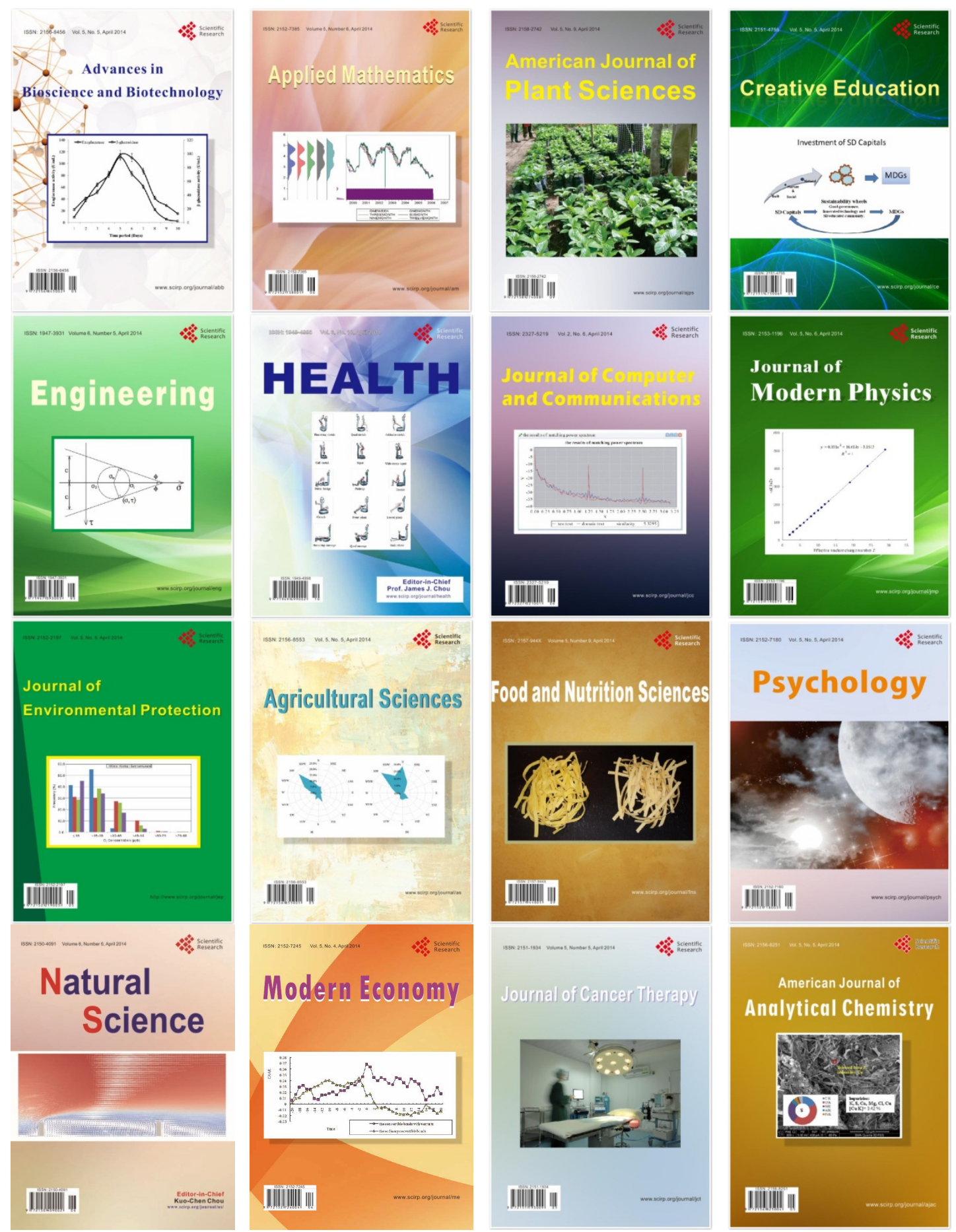